\title{
Roles of cysteines Cys115 and Cys201 in the assembly and thermostability of grouper betanodavirus particles
}

\author{
Chun-Hsiung Wang $\cdot$ Chi-Hsin Hsu $\cdot$ Yi-Min Wu • \\ Yu-Chun Luo $\cdot$ Mei-Hui Tu $\cdot$ Wei-hau Chang • \\ R. Holland Cheng $\cdot$ Chan-Shing Lin
}

Received: 1 March 2010/Accepted: 19 April 2010/Published online: 6 May 2010

(C) The Author(s) 2010. This article is published with open access at Springerlink.com

\begin{abstract}
The virus-like particle (VLP) assembled from capsid subunits of the dragon grouper nervous necrosis virus (DGNNV) is very similar to its native $T=3$ virion. In order to investigate the effects of four cysteine residues in the capsid polypeptide on the assembly/dissociation pathways of DGNNV virions, we recombinantly cloned mutant VLPs by mutating each cysteine to destroy the specific disulfide linkage as compared with thiol reduction to destroy all S-S bonds. The mutant VLPs of C187A and C331A mutations were similar to wild-type VLPs (WT-VLPs); hence, the effects of Cys187 and Cys331 on the particle formation and thermostability were presumably negligible. Electron microscopy showed that either C115A or C201A mutation disrupted de novo VLP formation significantly. As shown in micrographs and thermal decay curves, $\beta$-mercaptoethanol-treated WT-VLPs remained intact, merely resulting in lower tolerance to thermal disruption than native WT-VLPs. This thiol reduction broke disulfide linkages inside the pre-fabricated VLPs, but it did not disrupt the appearance of icosahedrons. Small dissociated capsomers from EGTA-treated VLPs were able to reassemble back to icosahedrons in the presence of calcium ions, but additional treatment with $\beta$-mercaptoethanol
\end{abstract}

C.-H. Wang · C.-H. Hsu · Y.-C. Luo · M.-H. Tu

C.-S. Lin $(\bowtie)$

Department of Marine Biotechnology and Resources, National

Sun Yat-sen University, Kaohsiung 80424, Taiwan, ROC

e-mail: shinlin@mail.nsysu.edu.tw

Y.-M. Wu · W. Chang

Institute of Chemistry, Academia Sinica, Taipei 11529, Taiwan,

ROC

R. H. Cheng

Department of Molecular and Cellular Biology, University

of California, Davis, CA 95616, USA during EGTA dissociation resulted in inability of the capsomers to reassemble into the icosahedral form. These results indicated that Cys115 and Cys201 were essential for capsid formation of DGNNV icosahedron structure in de novo assembly and reassembly pathways, as well as for the thermal stability of pre-fabricated particles.

Keywords Nervous necrosis virus - Virus-like particle . Disulfide linkages - Reassembly

\section{Introduction}

Piscine nodaviruses belong to the Betanodavirus genus in the Nodaviridae family that is a group of small, non-enveloped icosahedron viruses. More than 30 species of fish are infected by the betanodaviruses, which cause massive mortality in hatchery-reared larvae and juveniles [1]. The dragon grouper nervous necrosis virus (DGNNV), a betanodavirus, is a pathogen of Epinephelus lanceolatus. The viral genome is composed of two linear, single-stranded, positive-sensed RNAs. RNA1 ( $\sim 3.1 \mathrm{~kb})$ encodes an RNA-dependent RNA polymerase, and RNA2 $(\sim 1.4 \mathrm{~kb})$ encodes a capsid protein of $\sim 37 \mathrm{kDa}$, which can encapsulate the bipartite RNAs to form icosahedron particles [2,3]. The three-dimensional structures of DGNNV, malabaricus grouper nervous necrosis virus (MGNNV), and sea bass nodavirus (SBNV) virions have been elucidated by cryo-electron microscopy (Cryo-EM) [2, 4-6].

Several reports have demonstrated that disulfide linkage and calcium ion-mediated interactions between the capsid protein subunits are important for virion assembly [7-11]. In Simian virus 40 (SV40), the capsids can dissociate into VP1 pentamers in vitro in the presence of dithiothreitol (DTT) and ethylene glycol tetraacetic acid (EGTA) 
[8, 12-15]. Crystallographic analysis of SV40 confirms that the disulfide linkage between two Cys104 residues of neighboring subunits is a $\beta-\beta^{\prime}$ interpentameric interaction with two calcium ions bound per VP1 molecule [16, 17]. $\mathrm{X}$-ray structure analysis for insect nodaviruses reveals that intramolecular disulfide linkages in Nodamura virus are formed between Cys61 and Cys310 on the C-chain of coat protein (PDB ID: 1nov) [18]; similarly, two conserved cysteines are found in FHV (Cys69 and Cys318 in PDB ID: 2z2q), BBV (Cys69 and Cys318 in PDB ID: 2bbv), and $\mathrm{PaV}$ (Cys62 and Cys316 in PDB ID: 1f8v) [19-21]. However, the X-ray structure of fish nodavirus has not yet been determined.

The spontaneous assembly into DGNNV virus-like particle (VLP) from 180 copies of the capsid subunit that is recombinantly expressed in Escherichia coli [3] provides a tool to study the effects of cysteine mutations on the formation of viral icosahedron structure. In this study, we used site-directed mutagenesis and chemical reducing reagents to investigate the roles of four cysteines in virion assembly and dissociation. The effects of these mutations on the thermal stability of the VLPs were also investigated.

\section{Materials and methods}

Construction of cysteine mutants

Four cysteine residues (Cys115, Cys187, Cys201, and Cys331) in the DGNNV capsid protein were mutated to alanine by PCR-based site-directed mutagenesis. Cysteine mutants were constructed according to the manufacturer's protocol of the Quickchange II site-directed mutagenesis kit (Stratagene). Overlapping primer pairs corresponding to the four cysteines were used to introduce point mutations. The oligonucleotide pairs were designed as follows (mutation sites are underlined): C115A-F (5'-GAA ATT CAG CCA ATG GCC CCC GCA AAC ACG GGC-3'), C115A-R (5'-GCC CGT GTT TGC GGG GGC CAT TGG CTG AAT TTC-3'), C187A-F (5'-CGG CTG ATA CTC CTG GCT GTC GGC AAC AAC ACT-3'), C187A-R (5'-AGT GTT GTT GCC GAC AGC CAG GAG TAT CAG CCG-3'), C201A-F (5'-AAC GTG TCA GTG CTG GCT CGC TGG AGT GTT CGA-3'), C201A-R (5'-TCG AAC ACT CCA GCG AGC CAG CAC TGA CAC GTT$\left.3^{\prime}\right)$, C331A-F (5'-CCT GTT GGC ACT GTC GCC ACC AGG GTT GAC TCG-3'), and C331A-R (5'-CGA GTC AAC CCT GGT GGC GAC AGT GCC AAC AGG-3'). The plasmid pDA8 expressing the full-length DGNNV capsid protein was been cloned in our laboratory by Lu et al. [22] and VLPs could autonomously assemble in E. coli cells. Plasmid pDA8 (200 ng) was used as a template in 50- $\mu \mathrm{l}$ PCR reactions each containing $125 \mathrm{ng}$ primer,
$0.2 \mathrm{mM}$ dNTPs, and 2.5 U PfuUltra HF DNA polymerase. Cycling conditions were as follows: denaturation for $2 \mathrm{~min}$ at $95^{\circ} \mathrm{C}, 20$ cycles with denaturation for $30 \mathrm{~s}$ at $94^{\circ} \mathrm{C}$, annealing for $1 \mathrm{~min}$ at $59^{\circ} \mathrm{C}$, and extension for $4 \mathrm{~min} 45 \mathrm{~s}$ at $68^{\circ} \mathrm{C}$. Mutants included not only single site mutations but also other combinations of double, triple, and quadruple mutations among the four cysteine residues (Table 1). The base substitutions of all constructs were sequenced for confirmation. The cysteine-mutated capsid proteins were expressed in E. coli JM109 with isopropyl- $\beta$-D-thiogalactoside (IPTG) induction. Sodium dodecyl sulfate-polyacrylamide gel electrophoresis (SDS-PAGE) and western blotting were used to analyze the expression level and molecular weight of the cysteine-mutated capsid proteins.

\section{Electron microscopy}

The formation of cysteine-mutated VLPs was examined using sucrose density gradient centrifugation and electron microscopy as previously described [23]. Briefly, the E. coli cells expressing DGNNV capsid protein were harvested by centrifugation, and the cells were disrupted by a French press (Avestin Emulsiflex-C5). VLPs were purified by $10-40 \%$ sucrose gradient centrifugation. Samples of $5 \mu \mathrm{l}$ were applied to carbon-coated grids and left for $1 \mathrm{~min}$. After being washed twice with deionized water, the samples were stained with $2 \%$ uranyl acetate (UA; in Tris- $\mathrm{HCl}$, $\mathrm{pH}$ 8.0) for $40 \mathrm{~s}$. The grids were blotted with filter paper and dried in vacuo overnight. Specimens were examined using a 120-kV electron microscope (JEOL JEM-1400, equipped with a Gatan UltraScan 4000 CCD camera), and images were taken at a magnification of $40,000 \times$ with a defocus of $\sim 1.5 \mu \mathrm{m}$.

\section{Thermal stability of VLPs}

In order to analyze the stability of VLPs without disulfide linkages, $200 \mu \mathrm{g}$ of purified VLPs were treated with $10 \%$ $\beta$-mercaptoethanol in $10 \mathrm{mM}$ Tris- $\mathrm{HCl}(\mathrm{pH} \mathrm{8.0)}$, and thermal challenges were conducted at $40-85^{\circ} \mathrm{C}$ for $1 \mathrm{~h}$. Purified VLPs of C187A, C331A, and C187A/C331A (double mutant) cysteine mutants were also incubated at $40-85^{\circ} \mathrm{C}$ for $1 \mathrm{~h}$. The solutions were loaded onto a 5-ml 10-40\% sucrose density gradient centrifugation and fractionized as described elsewhere [23]. The remaining amounts of intact VLPs at fraction \#24 were employed to calculate the decay rates; therefore, the Arrhenius plot was used to find slopes for estimating the equivalent instability [24].

Dissociation and reassembly of VLPs

For in vitro dissociation studies, $200 \mu \mathrm{g}$ of purified VLPs were incubated with the chelating agent EGTA $(20 \mathrm{mM})$ or 
Table 1 Schematic representation of cysteine mutants on DGNNV capsid protein

a Amino acid residues are represented in the one-letter code and numbered according to the DGNNV capsid protein sequence (Genbank AAG22496)

b The formation of VLPs is listed at the right panel and indicated as "+"

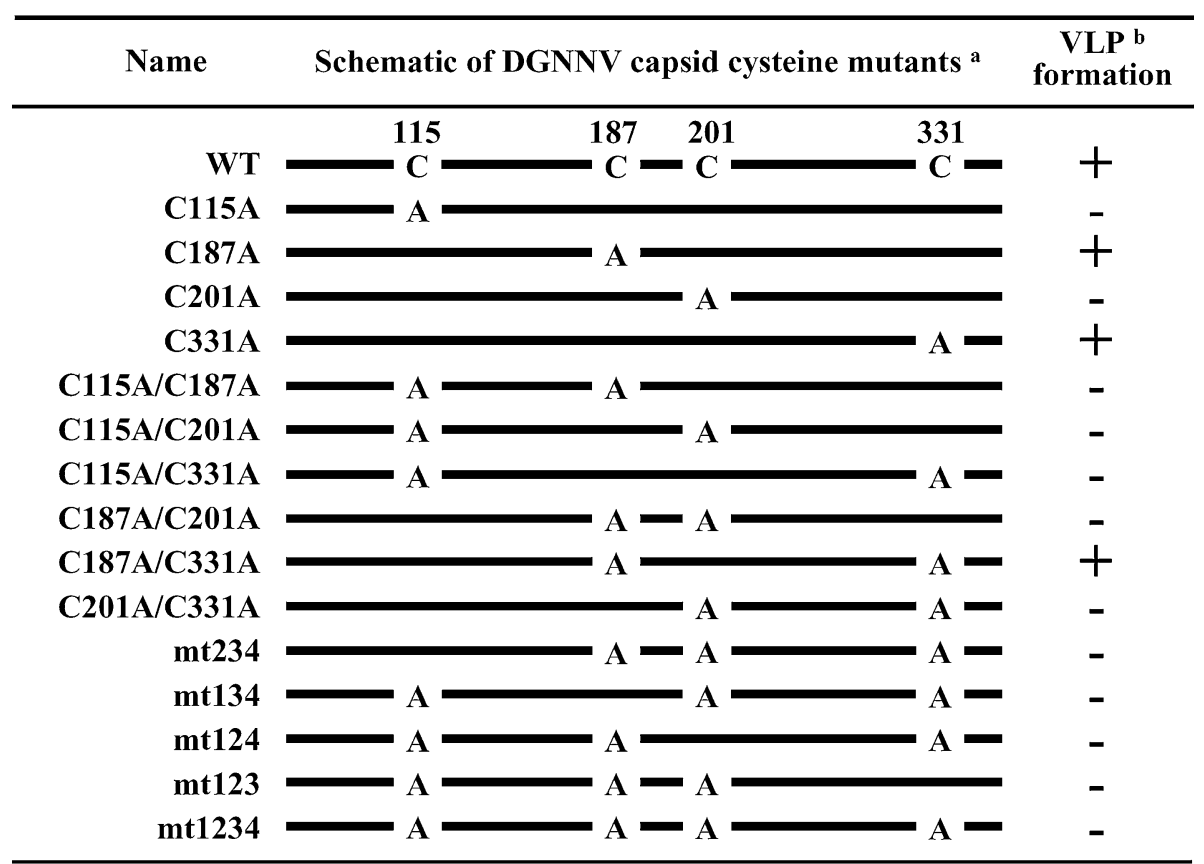

reducing agent $\beta$-mercaptoethanol (10\%) at $4{ }^{\circ} \mathrm{C}$ overnight [23]. For particle reassembly, the dissociated capsomers were incubated with $50-\mathrm{mM} \mathrm{CaCl}_{2}$ at $4{ }^{\circ} \mathrm{C}$ for $2 \mathrm{~h}$. In order to determine the ability of capsomers to form VLPs in the absence of disulfide bonds, dissociated capsomers were treated with $10 \% \beta$-mercaptoethanol at $4^{\circ} \mathrm{C}$ for $2 \mathrm{~h}$, and then incubated with $50-\mathrm{mM} \mathrm{CaCl}_{2}$ at $4^{\circ} \mathrm{C}$ for another $2 \mathrm{~h}$. After incubation, the mixtures were fractionated by $10-40 \%$ sucrose density gradient centrifugation and examined by electron microscopy. The absorbance was recorded continuously at $254 \mathrm{~nm}$, and the amount of reassembled VLPs in fraction \#24 was used to calculate the reassembly efficiency.

\section{Results}

The effects of cysteine mutations on de novo VLP formation

The DGNNV capsid protein contains four cysteine residues (Cys115, Cys187, Cys201, and Cys331) among its 338 amino acids (NCBI ID: AAG22496). In order to identify which cysteines are essential for disulfide bond formation as well as their effects on de novo VLP formation, the cysteines were substituted with alanines by site-directed mutagenesis. In addition to single site mutations for each cysteine, other combinations of double, triple, and quadruple mutations among the four cysteines were also acquired (Table 1). All recombinant proteins were expressed at high levels, as visualized in a Coomassie bluestained gel; further, western blotting analysis with antiVLP serum confirmed that the $37-\mathrm{kDa}$ bands represented the DGNNV capsid protein.

The ability of mutant capsid proteins to form VLPs intracellularly was analyzed using sucrose density gradient centrifugation and electron microscopy as previously described [23]. As a control, the profile of purified wildtype VLPs (WT-VLPs) in sucrose-gradient showed a major peak at the heavy fraction \#24, out of a total of 34 fractions (Fig. 1a; bold line: WT). The profiles for single C115A and single C201A mutants displayed a few peaks in the light fractions \#5 to \#15; but no peak at the fraction \#24 (Fig. 1a; thin line: C115A; dotted line: C201A). The profiles of the single C187A and double C187A/C331A mutants showed peaks of the light fractions from \#5 to \#15 and the heavy fraction at \#24 (Fig. 1b; thin line: C187A; dotted line: C187A/C331A). The C331A mutant demonstrates a major peak at fraction \#24 (Fig. 1b; bold line: C331A). There was no peak at fraction \#24 observed for the other double, triple, or quadruple cysteine mutants (data not shown).

As the fraction \#24 was negatively stained and observed by electron microscopy, WT-VLPs are $\sim 30 \mathrm{~nm}$ in diameter (Fig. 1d, WT), the same as native DGNNV virus (Fig. 1e). Electron microscopy examinations revealed that single $\mathrm{C} 115 \mathrm{~A}$ and single $\mathrm{C} 201 \mathrm{~A}$ mutants could not produce VLPs. The fraction \#24 from C187A or C331A mutants contained icosahedron particles (Fig. 1d, C187A and C331A) that were indistinguishable from the WT-VLPs 
(A)

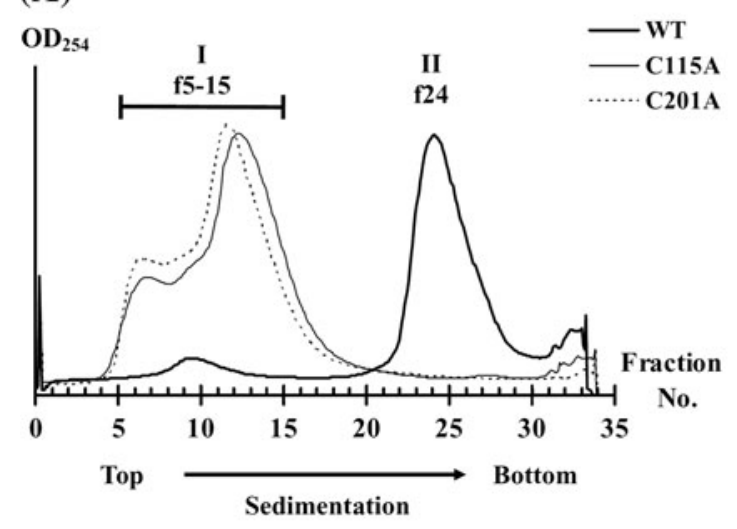

(C) Fractions \#5-\#15


(D) Fraction \#24
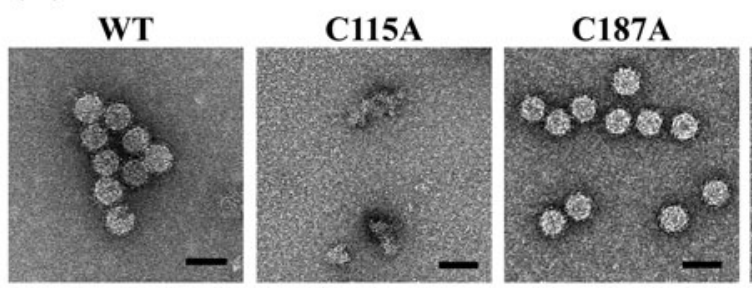
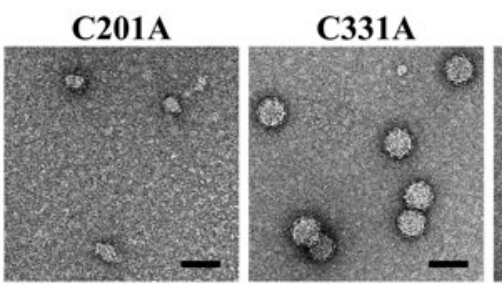

(B)

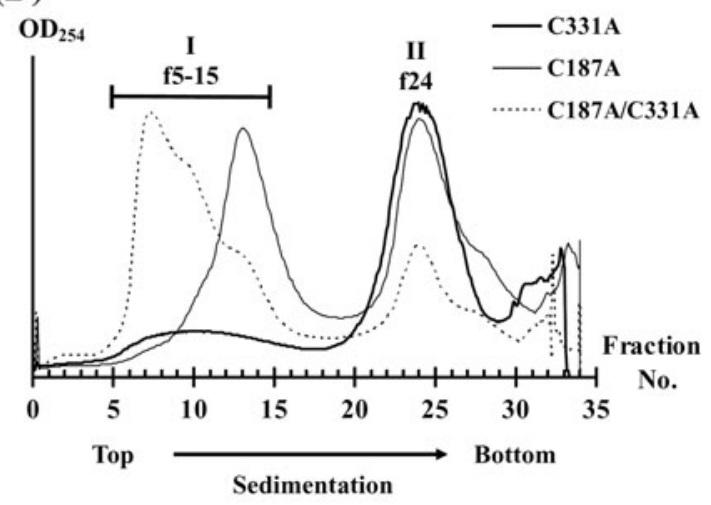

C201A

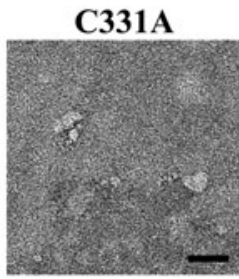

C187A/C331A

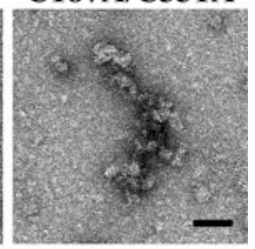

C187A/C331A

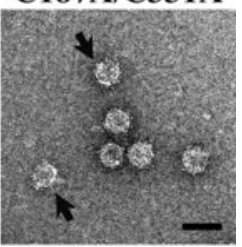

\section{(E) Control: native DGNNV virus}

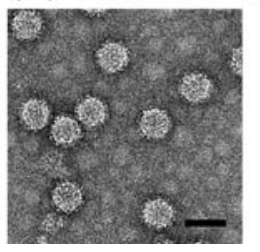

Fig. 1 VLP formations from the DGNNV capsid protein with cysteine mutations. a The intensity profiles of wild-type and cysteine mutants determined by sucrose density gradient centrifugation. Bold line: WT (no mutation); thin line: C115A; dotted line: C201A. b Bold line: $\mathrm{C} 331 \mathrm{~A}$; thin line: $\mathrm{C} 187 \mathrm{~A}$; dotted line: $\mathrm{C} 187 \mathrm{~A} / \mathrm{C} 331 \mathrm{~A}$. Fractions

(Fig. 1d, WT). A few defective particles of irregular shapes were observed in fraction \#24 of the C187A/C331A double mutant (arrows in Fig. 1d, C187A/C331A). The light fractions (\#5-\#15) in all mutants were composed of heterogeneous protein stains without a uniform shape (Fig. 2c). In summary, only the mutants with single C187A, single C331A, or double C187A/C331A produced VLPs; in other cases, the coat protein with a single mutation in either Cys115 or Cys201 could not de novo form VLPs. from \#5 to \#15 are denoted as I-f5-15 and fraction \#24 is denoted as II-f24. c, $\mathbf{d}$ Micrographs from fractions \#5 to \#15 and fraction \#24 of the cysteine mutants. The arrow indicates the broken particles with irregular shape. e Control: native DGNNV virus. Bar: $50 \mathrm{~nm}$

The effects of thiol reduction on pre-fabricated WT-VLPs

After the formation of icosahedron structure, the prefabricated WT-VLP particles with all four cysteines were soaked in the reducing or oxidizing reagents for $2 \mathrm{~h}$. Under electron microscopy, the conformation of VLPs remained intact whether in $\beta$-mercaptoethanol or diamide solution (data not shown). 

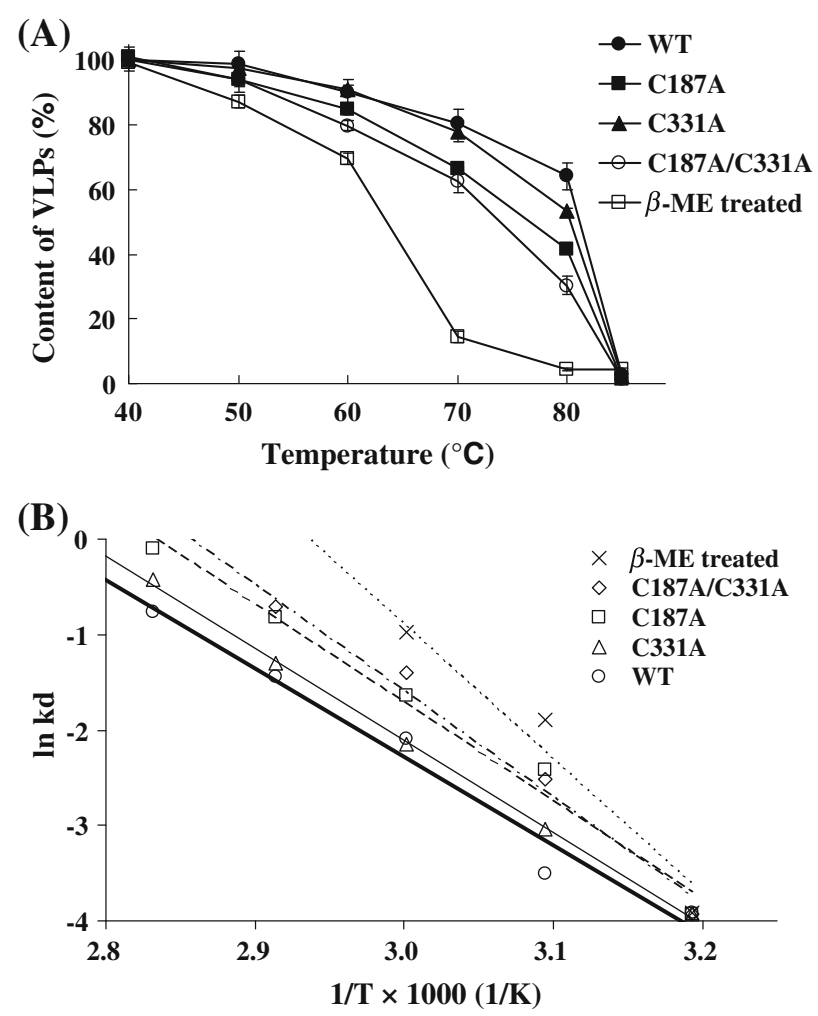

Fig. 2 Thermal stability of WT-VLPs, $\beta$-ME-VLPs, and cysteinemutant VLPs. a The amounts of VLPs in fraction \#24 were calculated for the treatments at $40-85^{\circ} \mathrm{C}$. b Arrhenius plots of the thermal decay rate constants for the VLPs

Although the particles under thiol reduction remained intact, the particles could be altered internally and they were unstable at high temperature. The pre-fabricated WT-VLPs were treated with $\beta$-mercaptoethanol and incubated at $40-85^{\circ} \mathrm{C}$ for $1 \mathrm{~h}$. As the profiles in sucrose-gradient centrifugation showed (Fig. 2a), when the temperature increased, the amounts of VLPs in the heavy fraction \#24 decreased in the range of $70-85^{\circ} \mathrm{C}$ for untreated WT-VLPs, but in the range of $60-70^{\circ} \mathrm{C}$ for $\beta$-mercaptoethanol-treated VLPs (Fig. 2a). As micrographs from electron microscopy showed VLPs were completely disrupted to amorphous aggregates or insoluble clumps when incubated with $\beta$-mercaptoethanol at temperatures higher than $70^{\circ} \mathrm{C}$ for $1 \mathrm{~h}$ (Fig. 3b), whereas unreduced WT-VLPs were not destroyed until $85^{\circ} \mathrm{C}$ (Fig. 3a). Thus, without the thermal challenge, the prefabricated WT-VLPs were not disrupted by the reducing agents and remained intact as did the untreated one; while $\beta$-mercaptoethanol treatment resulted in the thermal instability of the particles.

\section{Thermostability of mutant VLPs}

The mutants of C187A, C331A, and C187A/C331A could form VLPs (Fig. 1d); these mutant VLPs were also subjected to different temperatures for $1 \mathrm{~h}$ to evaluate their thermal stability. When the temperature approached the critical denaturation points, the amounts of particles in the heavy fraction \#24 decreased sharply (Fig. 2a). The critical denaturation temperature for $\mathrm{C} 187 \mathrm{~A}$ and $\mathrm{C} 331 \mathrm{~A}$ cysteine-mutant VLPs was between 70 and $85^{\circ} \mathrm{C}$, while the $\beta$-mercaptoethanol-treated WT-VLPs significantly decomposed at $60-70^{\circ} \mathrm{C}$.

Arrhenius plots of the decay constants for each mutant VLPs versus temperature reciprocals were used to determine the degrees of thermal instability (Fig. 2b). Activation energies of destabilization, which can be equivalent to the degrees of instability, were calculated from the multiplication of the Arrhenius slopes by the ideal gas constant $(1.987 \mathrm{cal} / \mathrm{mol} \mathrm{K}): 18.4$ for WT-VLPs, 20.4 for C187A, 19.2 for C331A, 22.2 for C187A/C331A, and 28.0 for $\beta$-mercaptoethanol-treated WT-VLPs. The C187Aand C331A-mutant VLPs were similar to WT-VLPs and much more stable than $\beta$-mercaptoethanol-treated particles.

The micrographs from electron microscopy in Fig. 3c show the characteristic quality of the particles; the C187A, C331A, and C187A/C331A VLPs remained intact after 1-h treatments at temperatures lower than $80^{\circ} \mathrm{C}$. Smear aggregates of insoluble protein complexes were observed at $85^{\circ} \mathrm{C}$ for the Cys187- and Cys331-mutant VLPs (Fig. 3c) and WT-VLPs (Fig. 3a).

In vitro dissociation and reassembly of VLPs

In the disassembly assay, the particles were dissociated by EGTA in the presence/absence of $\beta$-mercaptoethanol. As control, untreated particles were homogeneously intact (Fig. 4a, left panel). After EGTA treatment, the capsomers of irregular shapes were observed in negative staining electron microscopy (Fig. 4a, middle panel). This showed that VLPs could be dissociated into small capsomers by EGTA treatment alone [23].

In order to reverse the dissociation, calcium ions were added to the solution of dissociated capsomers to restore the particles. The small capsomers reassembled into homogeneous icosahedrons as observed under electron microscopy (Fig. 4a, right panel); their shapes and sizes are indistinct from the untreated VLPs (Fig. 4a, left panel).

However, after the $\beta$-mercaptoethanol treatment, the dissociated capsomers failed to reassemble to icosahedrons by $\mathrm{Ca}^{2+}$ (Fig. 4b); the reduction reaction could destroy all disulfide linkages in the capsid subunit. The micrographs from electron microscopy showed that mutant capsomers of C187A, C331A, and C187A/C331A could all reassemble into icosahedron particles (Fig. 4c); this is a pair of cysteine residues that were mutated to break partial disulfide linkages in the capsid. The 


\section{(A) WT-VLP}
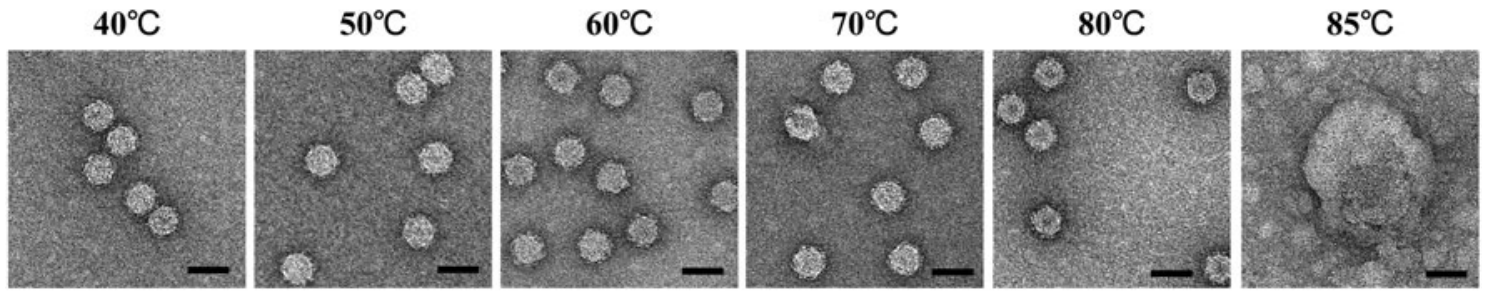

\section{(B) $\beta$-ME-treated}
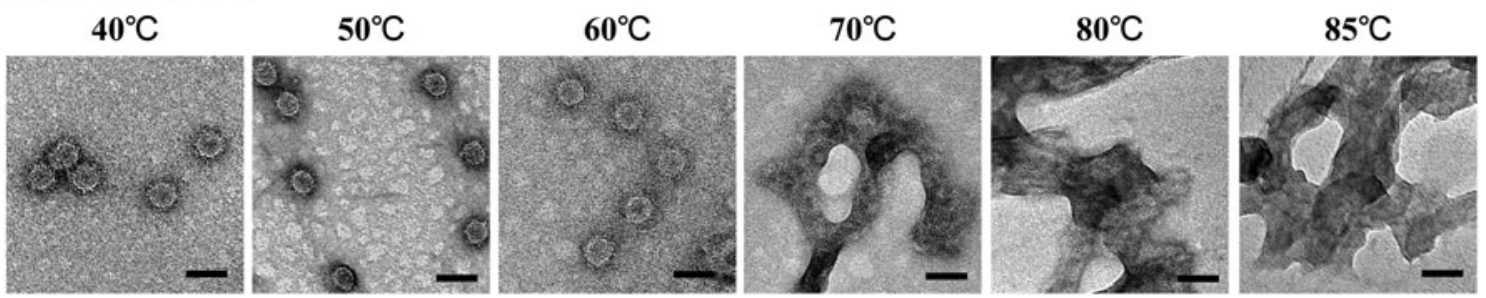

(C)

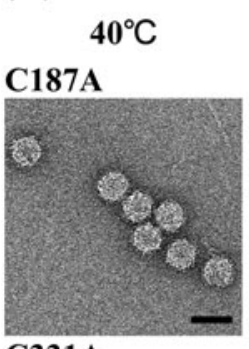

$50^{\circ} \mathrm{C}$

$60^{\circ} \mathrm{C}$

$70^{\circ} \mathrm{C}$

$80^{\circ} \mathrm{C}$

$85^{\circ} \mathrm{C}$
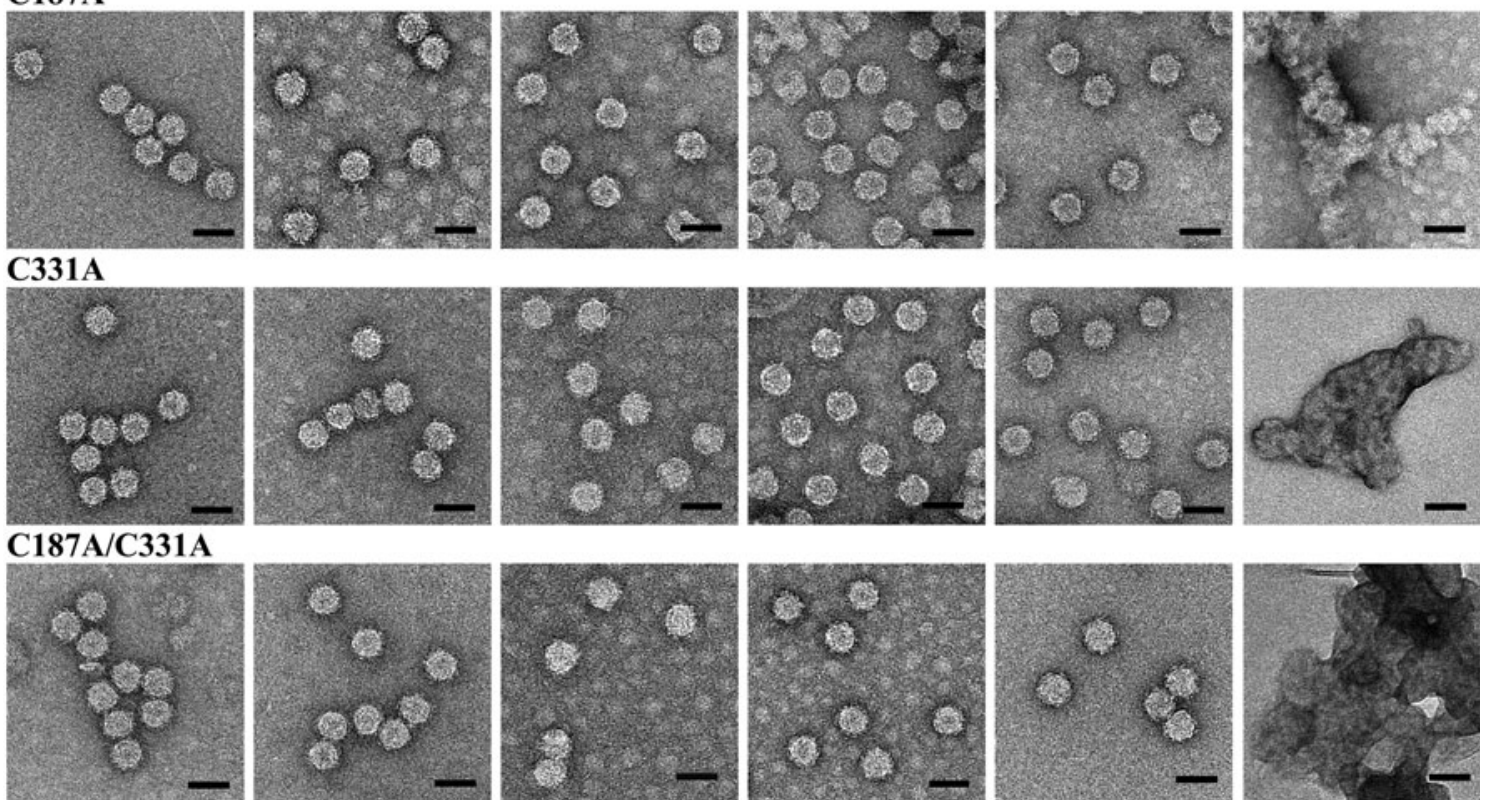

Fig. 3 Micrographs from electron microscopy of VLPs treated at different temperatures. a WT-VLPs; $\mathbf{b} \beta$-ME-VLPs; c cysteine-mutant VLPs. Bar: $50 \mathrm{~nm}$

reassembled C187A particles were UA-stain permeable, while reassembled $\mathrm{C} 187 \mathrm{~A} / \mathrm{C} 331 \mathrm{~A}$ particles exhibited some irregularities (Fig. 4c).

The VLP reassembly efficiencies of WT, C187A, $\mathrm{C} 331 \mathrm{~A}$, and $\mathrm{C} 187 \mathrm{~A} / \mathrm{C} 331 \mathrm{~A}$ were $97 \pm 1 \%, 95 \pm 2 \%$, $93 \pm 3 \%$, and $98 \pm 2 \%$, respectively, whereas $\beta$-mercaptoethanol-treated capsomers could not reassemble into VLPs.

\section{Discussion}

It has been demonstrated with a number of viruses that calcium and disulfide linkages are required for particle formation and virion stability [7, 8, 25]. Likewise, in the process of DGNNV particle formation, the DxxDxD motif in the 130th-135th residues is involved in the coordination of calcium ions [23] while the disulfide linkages present in 


\section{(A) WT-VLP}
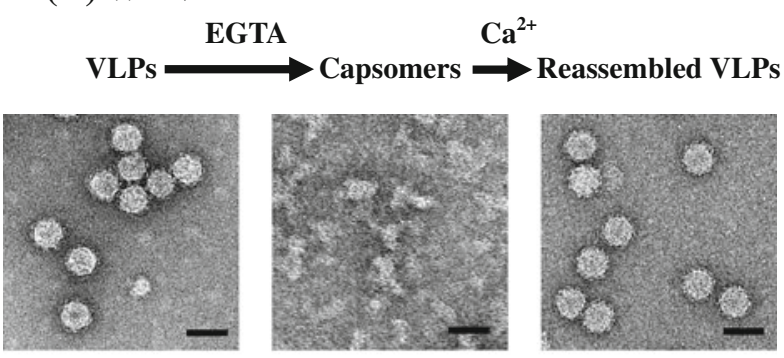

(B) WT-VLP $+\beta$-ME


(C) Mutant VLPs

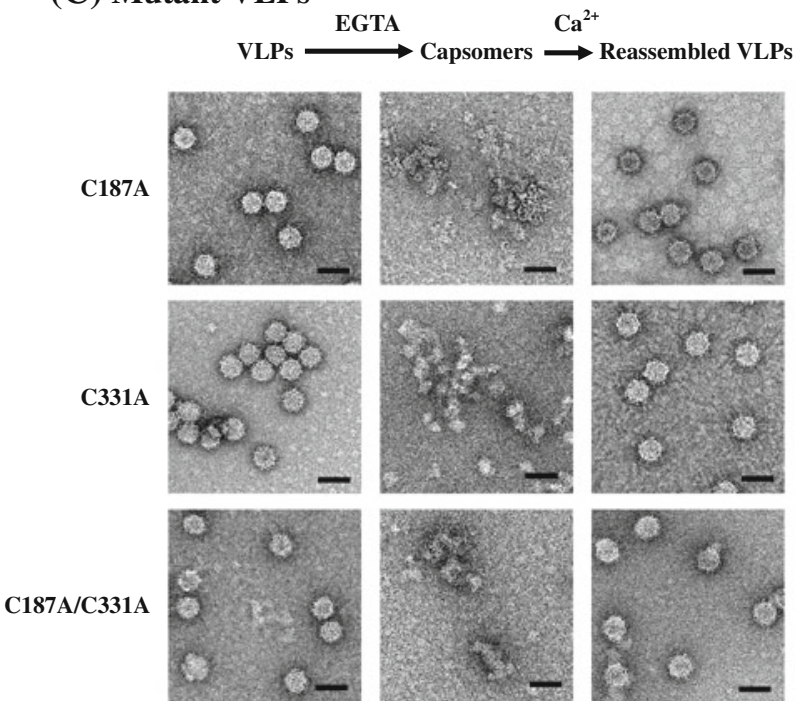

Fig. 4 Disassembly and reassembly of DGNNV VLPs. a WT-VLPs; b $\beta$-mercaptoethanol-treated WT-VLPs. c Reassembly of mutant VLPs of C187A, C331A, and C187A/C331A. Bar: $50 \mathrm{~nm}$

the DIEV capsid protein structure were intramolecular in a previous report [26]. This study further examined the roles of four cysteines in the DGNNV capsid protein for VLP formation and disassembly. Within the DGNNV capsid protein, all four cysteines were mutated to determine their roles in particle formation.

No icosahedron particles were observed for the single mutant of either C115A or C201A (Fig. 1d); the single mutation of these two cysteines disrupted the formation mechanism of VLPs. We also demonstrated that other cysteine mutants, except for C115A and C201A, retained the ability to form particles, similar to WT-VLPs (Fig. 1). These results suggest that the disulfide linkage of Cys115 and Cys201 residues likely exists and is required for de novo formation of VLP icosahedrons.

When VLP particles are pre-fabricated, the reducing agent cannot disrupt the VLP icosahedron structure (Fig. $3 \mathrm{~b}, \beta$-ME $40^{\circ} \mathrm{C}$ ). The thiol reduction only caused effects on the disulfide linkages inside the icosahedrons; electron microscopy (Fig. 3b) and Arrhenius plot analyses (Fig. 2b) revealed that $\beta$-mercaptoethanol-treated WT-VLPs had lower tolerances to thermal disruption than did the C187A and C331A mutants or untreated WT-VLPs. The mutant VLPs of two cysteines Cys 187 and Cys331 maintained the same efficiency in particle assembly and reassembly as WT-VLPs (Figs. 1, 4). Therefore, the effects of Cys187 and Cys331 mutations on VLP formation, in either de novo or reassembly pathway, are presumably negligible.

A paradox exists: the mutations in Cys115 and Cys201 resulted in no VLP formation, whereas the thiol reduction, mainly to break the presumable linkages of Cys 115 and Cys201, did not destroy pre-fabricated VLPs. This implies that once the particles are intact, the viral icosahedrons do not require an intramolecular S-S bond (including Cys115 and Cys201) to maintain their structural integrity, although their stability collapsed in response to thermal challenges.

Assembly and dissociation of DGNNV WT-VLPs can be reversed by adding calcium ions or EGTA, respectively (Fig. 4a) [23]. Once the disulfide linkages of Cys115 and Cys201 in dissociated capsomers were entirely disrupted by $\beta$-mercaptoethanol treatment, the resulting capsomers could not reassemble back to icosahedron particles (Fig. 4b); since the effects of Cys187 and Cys 331 could presumably be ignored. Furthermore, all mutant VLPs that carried mutations of $\mathrm{C} 115 \mathrm{~A}$ and $\mathrm{C} 201 \mathrm{~A}$ in their capsid protein could not de novo assemble into VLP icosahedrons. It can be inferred that destruction of the Cys115 and Cys201 linkage hindered icosahedron formation in the processes of de novo virion assembly (resulting from C115A and C201 mutations) and re-assembly post VLP dissociation (resulting from the treatment of EGTA and $\beta$-mercaptoethanol).

It can be hypothesized that two switches are present in DGNNV virion. First, the calcium ions switch between virion assembly and dissociation pathways reversibly. Second, the cellular reduction compounds switch particle capsid dissociating into capsomers irreversibly. Furthermore, the intramolecular Cys115-Cys201 disulfide bond is essential for properly de novo folding of the capsid subunits during icosahedron formation, and, in pre-fabricated VLP icosahedrons, the $\mathrm{S}-\mathrm{S}$ bond is not a critical factor for maintaining the icosahedron architecture which prevents the subunits from denaturation. The calcium ion 
concentration and the reducing condition in the cellular compartments or vesicles needs be further investigation to confirm the status of such switches at the time when particles dissociate in the early uncoating stage, or later when the virions assemble during the release process.

Acknowledgements We would like to thank the Cryo-EM Common Facility of Scientific Instrument Center, Academia Sinica, for electron microscopy operation. This work was partially supported by grants from the National Science Council, Taiwan (NSC96-2313-B-110002-MY3), and the Ministry of Education (97C031701, 96C031701). We also thank the reviewers and Dr. Simon White for proofreading the manuscript.

Open Access This article is distributed under the terms of the Creative Commons Attribution Noncommercial License which permits any noncommercial use, distribution, and reproduction in any medium, provided the original author(s) and source are credited.

\section{References}

1. B.L. Munday, J. Kwang, N. Moody, J. Fish Dis. 25, 127-142 (2002)

2. C.S. Lin, M.W. Lu, L. Tang, W. Liu, C.B. Chao, C.J. Lin, N.K. Krishna, J.E. Johnson, A. Schneemann, Virology 290, 50-58 (2001)

3. M.W. Lu, C.S. Lin, Arch. Virol. 148, 345-355 (2003)

4. L. Tang, C.S. Lin, K.K. Neel, M. Yeager, A. Schneemann, J.E. Johnson, J. Virol. 76, 6370-6375 (2002)

5. C.H. Wang, R.H. Cheng, C.S. Lin, The 108th General Meeting of American Society for Microbiology, Boston, MA, T-023 101 (2008)

6. X. Yan, K.A. Dryden, J. Tang, T.S. Baker, J. Struct. Biol. 157, 211-225 (2007)

7. P.L. Chen, M. Wang, W.C. Ou, C.K. Lii, L.S. Chen, D. Chang, FEBS Lett. 500, 109-113 (2001)
8. K.I. Ishizu, H. Watanabe, S.I. Han, S.N. Kanesashi, M. Hoque, H. Yajima, K. Kataoka, H. Handa, J. Virol. 75, 61-72 (2001)

9. P.S. Satheshkumar, G.L. Lokesh, V. Sangita, V. Saravanan, C.S. Vijay, M.R.N. Murthy, H.S. Savithri, J. Mol. Biol. 342, 10011014 (2004)

10. M.B. Sherman, R.H. Guenther, F. Tama, T.L. Sit, C.L. Brooks, A.M. Mikhailov, E.V. Orlova, T.S. Baker, S.A. Lommel, J. Virol. 80, 10395-10406 (2006)

11. R. Zhao, A.T. Hadfield, M.J. Kremer, M.G. Rossmann, Virology 227, 13-23 (1997)

12. J.N. Brady, J.D. Kendall, R.A. Consigli, J. Virol. 32, 640-647 (1979)

13. J.N. Brady, V.D. Winston, R.A. Consigli, J. Virol. 23, 717-724 (1977)

14. J.N. Brady, V.D. Winston, R.A. Consigli, J. Virol. 27, 193-204 (1978)

15. M.C. Colomar, C. Degoumois-Sahli, P. Beard, J. Virol. 67, 2779 2786 (1993)

16. R.C. Liddington, Y. Yan, J. Moulai, R. Sahli, T.L. Benjamin, S.C. Harrison, Nature 354, 278-284 (1991)

17. T. Stehle, S.J. Gamblin, Y. Yan, S.C. Harrison, Structure 4, 165 182 (1996)

18. A. Zlotnick, P. Natarajan, S. Munshi, J.E. Johnson, Acta Crystallogr. D 53, 738-746 (1997)

19. A.J. Fisher, B.R. McKinney, A. Schneemann, R.R. Rueckert, J.E. Johnson, J. Virol. 67, 2950-2953 (1993)

20. L. Tang, K.N. Johnson, L.A. Ball, T. Lin, M. Yeager, J.E. Johnson, Nat. Struct. Biol. 8, 77-83 (2001)

21. J.P. Wery, V.S. Reddy, M.V. Hosur, J.E. Johnson, J. Mol. Biol. 235, 565-586 (1994)

22. M.W. Lu, W. Liu, C.S. Lin, J. Gen. Virol. 84, 1577-1582 (2003)

23. Y.M. Wu, C.H. Hsu, C.H. Wang, W. Liu, W.H. Chang, C.S. Lin, Arch. Virol. 153, 1633-1642 (2008)

24. A. Erarslan, H. Koçer, J. Chem. Technol. Biotechnol. 55, 79-84 (1992)

25. J. Paintsil, M. Müller, M. Picken, L. Gissmann, J. Zhou, J. Gen. Virol. 79, 1133-1141 (1998)

26. J.V. Krondiris, D.C. Sideris, J. Gen. Virol. 83, 2211-2214 (2002) 